\title{
CALIDAD DE LA CANAL DEL POTRO GALLEGO DE MONTE
}

\author{
CARCASS QUALITY OF GALICIAN MOUNTAIN FOALS
}

Franco, D. ${ }^{1}$, Fernández, M. ${ }^{1}$, Temperán, S. ${ }^{1}$, García, L. ${ }^{1}$ y Lorenzo, J.M. ${ }^{1 *}$

${ }^{1}$ Centro Tecnológico de la Carne. San Cibrao das Viñas. Ourense. Spain. *jmlorenzo@ceteca.net

Palabras CLAVE ADICIONALES

Despiece. Morfometría. Sistema de explotación.

\section{RESUMEN}

Fueron estudiadas las características de la canal de 50 potros Gallego de Monte provenientes de tres explotaciones. El peso al sacrificio de los potros de diez meses de la explotación extensiva acabados con heno y pienso ecológico (SEC) fue superior $(p<0,001)$ a los de la explotación extensiva, de un año, sin acabado (SE) (199 vs. 138 kg). El rendimiento canal también se vio afectado (53,35\% vs. 49,48\%, p<0,01). El porcentaje del cuarto delantero fue superior $(p<0,001)$ en los animales criados en libertad (SEL, 39,59\%) que en SE $(38,66 \%)$ y SEC $(35,76 \%)$. El porcentaje de magro y la grasa total fueron superiores en SEC $(p<0,001)$.

\section{SUMMARY}

The carcass characteristics of 50 Galician mountain foals from three different farms were studied. Slaughter weight was higher $(p<0.001)$ in foals, ten months old, from SEC vS. SE a year old (199 vs. $138 \mathrm{~kg}$ ). Dressing percentage was also affected (53.35\% vs. $49.48 \%, p<0.01)$. The percentage of front quarter was higher $(p<0.001)$ in SEL (39.59\%) than SE (38.66\%) and SEC (35.76\%). Nevertheless, the lean percentage and total fat was significantly higher in SEC foals (73 vs. 69 $p<0.05 ; 6.68$ vs. $3.25, p<0.001$ ).

\section{INTRODUCCIÓN}

Aunque su consumo es marginal res-

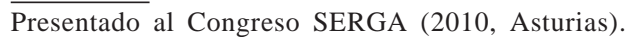
Financiación: Consellería do Medio Rural de la Xunta de Galicia (Proyecto FEADER 2007-03). Las cooperativas Monte Cabalar y Sabucedo Puxa y el Centro de Investigaciones Agrarias de Mabegondo proporcionaron las canales.

\author{
AdDitiONAL KEYWORDS \\ Carcass cutting. Morphometry. Production system.
}

pecto al de ternera, pollo o cerdo (LombardiBoccia et al., 2005), la carne de caballo presenta ventajas como rendimiento a la canal cercano al 70\% (Sarries y Beriain, 2005; Lanza et al., 2009) y una excelente calidad nutricional, con bajo contenido graso, rico en hierro hemo, bajo colesterol y un perfil de ácidos grasos con altos niveles de insaturados y vitamina B (Badiani et al., 1997; Lorenzo et al., 2010).

El caballo Gallego de Monte es una denominación de la población equina explotada en régimen de libertad en los montes de Galicia relacionada directamente con el caballo de Pura Raza Gallega, con el tronco Celta en común, y puede considerarse como una agrupación racial en fase de estudio que conduzca a un futuro reconocimiento oficial, cuyo censo se estima en más de 20000 cabezas (Fernández et al., 2001). No hay en la bibliografía medidas morfométricas ni escandallos de la canal de este recurso zoogenético. El presente trabajo tiene como objetivo estudiar las características de la canal de potro Gallego de Monte, como primer paso para evaluar este recurso no explotado en su totalidad en Galicia con una orientación productiva cárnica.

\section{MATERIAL Y MÉTODOS}

Se han utilizado 50 animales, provenientes de tres explotaciones: sistema extensivo en régimen de libertad (SEL) en los montes de Sabucedo (Pontevedra, España), donde 
los animales, de gran rusticidad, están muy adaptados a su medio ambiente, aguantando condiciones extremas tanto de alimentación como climatológicas en un sistema de curros; sistema de explotación extensivo (SE), con animales provenientes de la Asociación Monte Cabalar de A Estrada (Pontevedra, España) que es un sistema extensivo vallado, donde el animal está sometido a un mayor grado de manejo, por parte de los dueños (tratamientos veterinarios, control de pesos, etc...); y por último, sistema de explotación extensiva con acabado (SEC), con animales del rebaño experimental del Centro de Investigaciones Agrarias de Mabegondo, que han permanecido con sus madres en pastoreo, a excepción de los últimos tres meses que fueron alimentados con heno y pienso ecológico. Para los animales SEL no fue posible obtener el peso vivo. Los animales fueron sacrificados en un matadero comercial (A Estrada, Pontevedra). Las canales fueron pesadas y enfriadas a $4^{\circ} \mathrm{C}$ en cámara refrigerada durante 24 horas. Sobre la canal izquierda (planta piloto del Centro Tecnológico de la Carne) en condiciones refrigeradas se tomaron medidas morfométricas (tabla I) descritas por De Boer et al. (1974) y Carballo et al. (2000). También se calculó el índice de compacidad de canal (peso/longitud) y el índice de compacidad de la pierna (longitud/anchura)(Espejo et al., 2000). El escandallo de la media canal izquierda se realizó según la metodología de Carballo et al. (2000) en vacuno, obteniéndo 19 cortes comerciales (tabla II). Los resultados se analizaron mediante ANOVA con el programa SPSS 18.0 considerando el efecto sistema de explotación. Se aplicó el test de Duncan cuando la diferencia entre medias era significativa.

\section{RESULTADOSYDISCUSIÓN}

En la tabla I se presenta la morfología y principales características de la canal de los animales de estudio. Se encontraron diferencias $(p<0,001)$ en el peso al sacrificio para los animales de SEC frente a SE (199 vs. $138 \mathrm{~kg}$ ) a pesar de que los primeros se sacrificaron a los diez meses y medio mientras los segundos tenían un año de edad. El

Tabla I. Rendimiento y morfología de la canal de potros gallegos de monte. Efecto del sistema de explotación. (Dressing percentage and carcass morphology, of Galician mountain foals. Effect of livestock production system).

Características canal Extensivo-libertad SEL Extensivo SE Extensivo-acabado SEC SEM Sig

\begin{tabular}{|c|c|c|c|c|c|}
\hline Animales & 14 & 16 & 20 & & \\
\hline Edad & $212 \pm 49^{a}$ & $369 \pm 8^{b}$ & $313 \pm 42 \mathrm{c}$ & 10 & *** \\
\hline Peso vivo & - & $138 \pm 20$ & $199 \pm 29$ & 6,45 & *** \\
\hline Peso canal & $66,25 \pm 21,32^{\mathrm{a}}$ & $68,36 \pm 9,69^{a}$ & $119,24 \pm 34,98^{b}$ & 4,9 & *** \\
\hline Rendimiento canal & - & $49,48 \pm 4,83$ & $53,35 \pm 1,73$ & 0,69 & ** \\
\hline \multicolumn{6}{|l|}{ Morfología de la canal (cm) } \\
\hline Longitud canal & $82,50 \pm 10,79^{a}$ & $89,23 \pm 3,25^{b}$ & $95,02 \pm 5,40 \mathrm{c}$ & 1,17 & *** \\
\hline Longitud pierna & $62,60 \pm 5,44^{\mathrm{a}}$ & $64,97 \pm 2,18^{a}$ & $68,07 \pm 2,90^{\mathrm{b}}$ & 0,58 & *** \\
\hline Espesor pierna & $12,28 \pm 2,34^{\mathrm{a}}$ & $13,14 \pm 0,67^{\mathrm{a}}$ & $16,65 \pm 1,42^{b}$ & 0,34 & *** \\
\hline Perímetro pierna & $70,92 \pm 9,97^{a}$ & $69,76 \pm 2,90^{\mathrm{a}}$ & $81,95 \pm 5,16^{b}$ & 1,18 & *** \\
\hline Profundidad externa pecho & $46,71 \pm 6,76^{a}$ & $45,79 \pm 1,87^{\mathrm{a}}$ & $49,72 \pm 2,71^{b}$ & 0,60 & * \\
\hline Profundidad interna pecho & $30,28 \pm 3,07^{a}$ & $30,76 \pm 1,57^{a}$ & $32,92 \pm 1,89^{b}$ & 0,34 & ** \\
\hline$\| \mathrm{C}$ & $0,79 \pm 0,19^{a}$ & $0,76 \pm 0,09^{a}$ & $1,25 \pm 0,35^{b}$ & 0,04 & $\star \star *$ \\
\hline ILTP & $5,22 \pm 0,76^{a}$ & $4,95 \pm 0,21^{\mathrm{a}}$ & $4,11 \pm 0,32^{b}$ & 0,09 & *** \\
\hline
\end{tabular}

${ }^{* * *} p<0,001,{ }^{* *} p<0,01,{ }^{*} p<0,05$ 


\section{CALIDAD DE LA CANAL DEL POTRO GALLEGO DE MONTE}

Tabla II. Principales cortes comerciales y composición tisular de la canal de potros Gallegos de monte. Efecto del sistema de explotación. (Main commercial meat cut and tisular composition of Galician mountain foals. Effect of livestock production system).

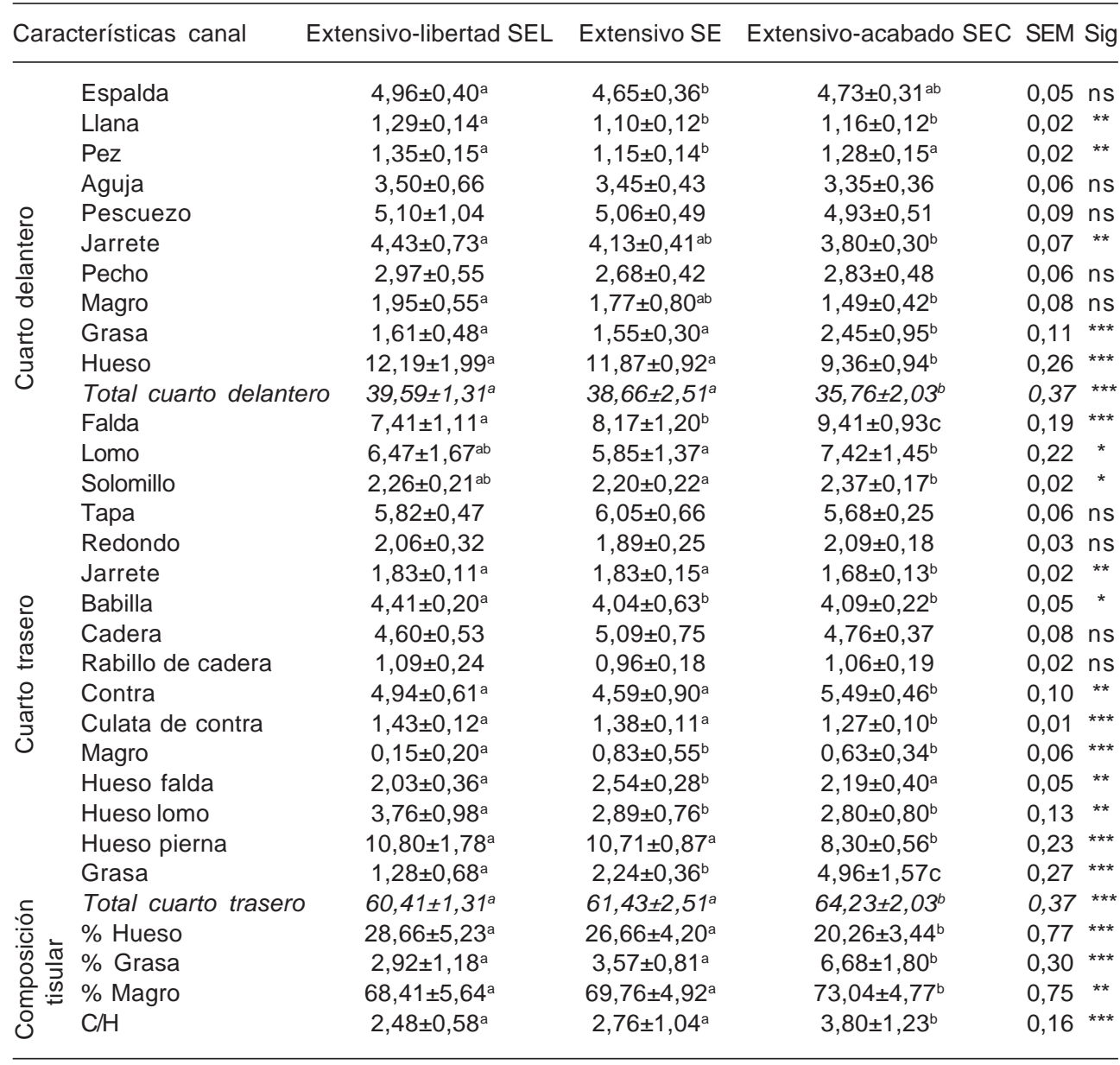

${ }^{* * \star p} p<0,001,{ }^{*} p<<0,01,{ }^{*} p<0,05$, ns: no significativo.

acabado afectó al rendimiento canal entre los animales acabados y aquellos sin acabar (53,35\% vs. 49,48\%, $\mathrm{p}<0,01)$, así como a todas las medidas lineales e índices de compacidad de la canal y de la pierna (tabla I). Si bien el rendimiento canal se incrementa con la edad del animal (Sarries y Beriain, 2005) en este caso el acabado fue más importante en el peso vivo y el rendimiento canal. Los pesos canal y los rendimientos están muy alejados de los obtenidos en otros potros sacrificados a mayor edad (16-24 meses de edad) y con razas de aptitud cárnica como Burguete (Sarries y Beriain, 2005 y HispanoBretón (Juárez et al., 2009). El rendimiento canal de potros Burguete pueden llegar hasta un 67\% (Sarries y Beriain, 2005).

El despiece de la media canal izquierda y la composición tisular se muestra en la tabla II, no hay en la bibliografía datos relativos 
al despiece de canales equinas, por lo que no es posible comparar con otras razas. En el cuarto delantero, el porcentaje de peso fue significativamente superior en los animales en régimen de libertad frente a los acabados con pienso, lo cual puede ser indicativo de su mayor rusticidad (39,59\%> 38,66>35,76\% para SEL, SE y SEC respectivamente); no se encontraron diferencias en el porcentaje de trasero (tabla II).

El porcentaje de magro y grasa total fue mayor en los animales finalizados con pienso (73 vs. $69 \mathrm{p}<0,05 ; 6,68$ vs. 3,25, $\mathrm{p}<0,001)$ que presentaron menor porcentaje de hueso, siendo la relación carne/hueso superior en los animales finalizados (3,8 vs. 2,62). No hay en la bibliografía datos relativos al despiece de canales, por lo que no es posible hacer comparaciones, debido a la edad de sacrificio y raza y tampoco es el objeto de este estudio, pero si se puede comprobar que

\section{BIBLIOGRAFÍA}

Badiani, A., Nanni, N., Gatta, P., Tolomelli, B. and Manfredini, M. 1997. Nutrient profile of horsemeat. J. Food Compos. Anal., 10: 254269.

Carballo, J.A., Monserrat, L. y Sánchez, L. 2000. Composición de la canal bovina. En: Metodología para el estudio de la calidad de la canal y de la carne en rumiantes. Monografía INIA: Serie Ganadera, 1: 107-123.

De Boer, H., Dumont, B.L., Pomeroy, R.W. and Weniger, J.H. 1974. Manual on E.A.A.P. reference methods for the assessment of carcass characteristics in cattle. Livest Prod. Sci., 1: 151-164.

Espejo, M., García, S., López, M.M., Izquierdo, M., Robles, A. y Costela, A. 2000. Morfología de la canal bovina. En: Metodología para el estudio de la calidad de la canal y de la carne. Monografía INIA: Serie Ganadera, 1: 69-80.

Fernández, M., Rivero, G., Alonso, M., Rivero, C.J., Pose, H., Justo, J.R., Adán, S., Díaz, R., Rois, D. y Carril, J.A. 2001. Razas autóctonas de Galicia en peligro de extinción. Servicio de Estudios y Publicaciones de la Consellería de Política Agroalimentaria e Desenvolvemento Rural. las canales se caracterizan por tener un alto contenido en carne, mayor en aquellos animales finalizados $(73,0$ vs. 69,09) y superior al 68,41 mostrado por Znamirowska (2005) en caballos de 6-10 años. Por el contrario los porcentajes de las piezas nobles como solomillo y lomo, así como el sumatorio de piezas que engloba la carne de primera no fueron afectados por el sistema de explotación.

En conclusión, el acabado tuvo un efecto positivo sobre el peso y rendimiento de la canal, aun en animales de menor edad y sobre el porcentaje de trasero que contiene las piezas de mayor valor. Los porcentajes de magro y grasa fueron superiores en los animales finalizados con concentrado. Es necesario un mejor manejo para aumentar el rendimiento cárnico, sin olvidar el papel de este ganado en el control de vegetación en los montes de Galicia que justifica su conservación y propagación.

Xunta de Galicia. Santiago de Compostela. La Coruña. España.

Juárez, M., Polvillo, O., Gómez, M.D., Alcalde, M.J., Romero, F. and Varela, M. 2009. Breed effect on carcass and meat quality of foals slaughtered at 24 months of age. Meat Sci., 83: 224-228.

Lanza, M., Landi, C., Scerra, M., Galofaro, V. and Pennisi, P. 2009. Meat quality and intramuscular fatty acid composition of Sanfratellano and Haflinger foals. Meat Sci., 81: 142-147.

Lombardi-Boccia, G., Lanzi, S. and Aguzzi, A. 2005. Aspects of meat quality: trace elements and $B$ vitamins in raw and cooked meats. J. Food Compos. Anal., 18: 39-46.

Lorenzo, J.M., Fuciños, C., Purriños, L. and Franco, D. 2010. Intramuscular fatty acid composition of "Galician Mountain" foals breed. Effect of sex, slaughtered age and livestock production system. Meat Sci. (In press).

Sarriés, M.V. and Beriain, M.J. 2005. Carcass characteristics and meat quality of male and female foals. Meat Sci., 70: 141-152.

Znamirowska, A. 2005. Prediction of horse carcass composition using linear measurements. Meat Sci., 69: 567-570. 